\title{
Coordinated frequency regulation by offshore wind farms and VSC-HVDC transmission
}

\author{
Hongzhi Liu*, Zhe Chen \\ Department of Energy Technology, Aalborg University, DK-9220 Aalborg, Denmark
}

\begin{abstract}
With the increasing wind penetration, large-scale offshore wind farms exert significant impact on power system security and operation, and thus are required to contribute to system frequency regulation. This paper develops a coordinated control strategy for offshore wind farms with voltage source converter-based HVDC (VSC-HVDC) transmission system to participate in power system frequency regulation. The strategy explores the frequency support capability of offshore wind farms and VSC-HVDC. By implementing the proposed coordinated control, the VSCHVDC link is able to provide quick virtual inertial response to onshore grid frequency drops. Shortly afterwards, the offshore wind farm detects the frequency changes and start to participate in inertial response and primary frequency control. The offshore wind farms, in this study, consist of full-converter wind turbines (FCWTs). Simulation results are presented to demonstrate the frequency regulation capability of VSC-HVDC and validate the effectiveness of the proposed coordinated control strategy.
\end{abstract}

Keywords: Frequency regulation, inertial response, offshore wind farm, primary frequency control, VSC-HVDC

\section{Introduction}

Power system frequency stability refers to the ability of a power system to maintain stable frequency following a severe system upset resulting in a significant imbalance between generation and load [1]. In the event of a sudden loss of a large power supply or a sudden increase in power demand, synchronous generators are able to extract kinetic energy (inertia response) and provide primary control reserve to stabilize the system frequency. As offshore wind farms are displacing conventional power plants, it is also necessary for wind turbines to contribute to frequency regulation like synchronous generators. Offshore wind farms, in general, consist of variable-speed wind turbines with power electronic converters. These turbines are naturally inertia-less due to the decoupling of converters [2] and have no primary control reserve as the wind is not controllable [3]. Yet, efforts have been made to allow the converter-interfaced wind turbines to inject their rotational kinetic energy into the grid during frequency deviation by converter controls [4]. Energy reserve margin has also been achieved by operating the turbines away from their optimal points at the expense of less generation [5], [6].

To integrate large-scale offshore wind farms into the onshore power grid, VSC-HVDC transmission is more attractive and applicable compared to HVAC transmission system. For transferring the same amount of power, DC cables have lower losses and have no limitations in length owing to the immunity from charging current [7], [8]. VSC-HVDC also has other advantages such as fully controlled power flow and independent and rapid control of active and reactive power [7], [8]. However, offshore wind farms would not be directly affected by onshore system disturbances due to the decoupling of VSC-HVDC. The decoupling would prevent offshore wind farms from immediate responding to the frequency excursion of onshore AC network. As for offshore wind applications, the contribution of VSC-HVDC to power system frequency regulation was investigated in [9]. However, all these studies focused on developing artificial

\footnotetext{
* Manuscript received June 15, 2013; revised July 30, 2013.

Corresponding author. Tel.: +45-50114828; E-mail address: hli@et.aau.dk.
} 
coupling methods between offshore and onshore networks using VSC-HVDC, but the VSC-HVDC itself did not participate in the actual frequency regulation.

This study addresses the frequency regulation contribution of both VSC-HVDC transmission system and offshore wind farms. A new coordinated control strategy is developed which enables VSC-HVDC to participate in power system frequency regulation. In addition, under the proposed control, all offshore wind turbines would respond to the onshore frequency excursion timely.

The organization of this paper is as follows. Section 2 introduces the FCWT model and its frequency regulation approaches. Section 3 addresses the frequency regulation capability of VSC-HVDC and the proposed coordinated frequency control strategy. Section 4 presents a series case studies and simulation results. Section 5 presents the conclusions of this study.

\section{Wind Turbine Model and Control Design}

\subsection{Wind turbine model}

The general configuration and control scheme of the FCWT along with its operation curve adopted in this paper is shown in Fig. 1. The generator-side converter controls the generator active power and maintains the generator stator voltage to its rated value. At the same time, the grid-side converter controls the DC-link voltage and the reactive power flow to the offshore AC grid. In the traditional control strategy [10], the active power reference $P_{\text {ref }}$ is only provided by a maximum power point tracking (MPPT) approach and the wind turbine generator will not respond to grid frequency deviation. However, to implement frequency support, an ancillary frequency control loop [4], [6] is applied to wind turbines as illustrated in Fig. 2.

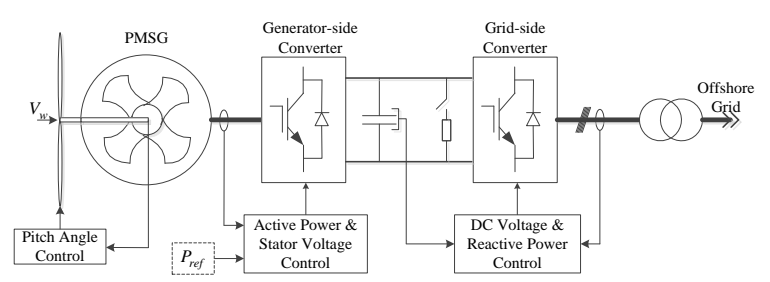

(a)

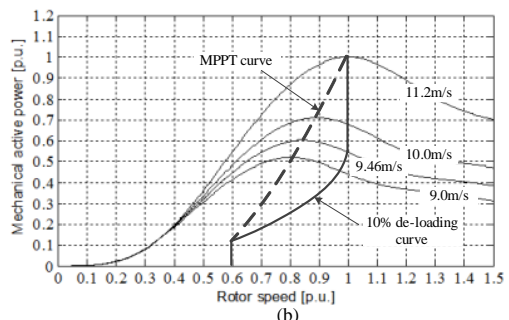

(b)

Fig. 1. Scheme of FCWT and its operating curves: (a) configuration and (b) operation curves.

\subsection{Ancillary control for frequency regulation}

The ancillary frequency regulation control of the FCWT shown in Fig. 2 consists of two components. The component $P_{\text {iner }}$ is proportional to the derivative of the grid frequency, emulating the inertial response of a synchronous generator. The component $P_{\text {prim }}$ is proportional to the absolute deviation of the grid frequency, emulating the primary frequency control of the governor of a conventional synchronous generator.

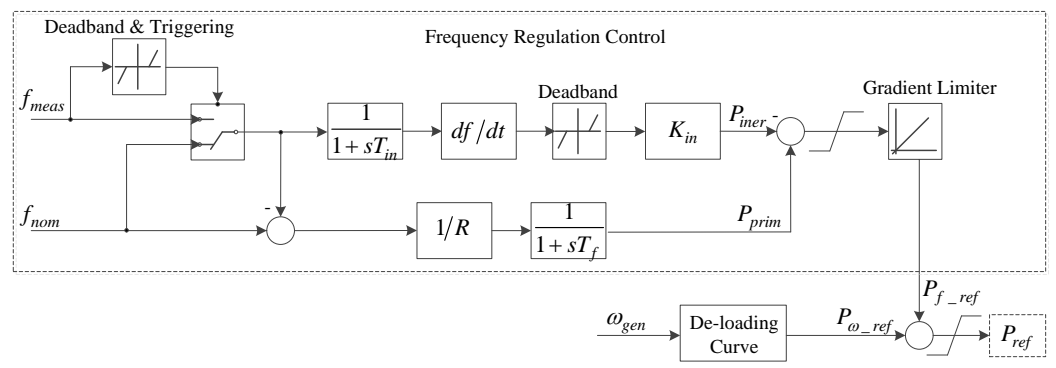

Fig. 2. Ancillary frequency control loop for FCWTs.

To enable the FCWT to provide primary reserve, a de-loading power tracking operation [11], [12] is applied to wind turbines instead of MPPT. Fig. 1 (b) compares the MPPT curve with pre-defined $10 \%$ deloading power curve that is adopted in this study. As shown, when the wind speed is below $9.46 \mathrm{~m} / \mathrm{s}$, the 
over-speed operation of the generator rotor is able to reduce the turbine generation to $90 \%$ of the available power. However, since the rotor speed may not be allowed to exceed $1.0 \mathrm{pu}$, it is obvious that only the over-speed operation cannot provide the pre-defined power reserve when the wind speed is above a certain value $(9.46 \mathrm{~m} / \mathrm{s}$ in this paper). Therefore, when the wind speed ranges from $9.46 \mathrm{~m} / \mathrm{s}$ to $11.2 \mathrm{~m} / \mathrm{s}$, the pitch angle controller is also activated to decrease the generator power. When the wind speed is above $11.2 \mathrm{~m} / \mathrm{s}$, the turbine rotor operates at its rated speed even in normal operations and the over-speed operation is not possible. In this case, the de-loading is realized by increasing the pitch angle only.

\section{VSC-HVDC Model and Control Design}

In this study, a $\pm 150 \mathrm{KV}$ VSC-HVDC transmission link is used to connect offshore wind farms to the onshore AC grid as shown in Fig. 3. In normal operations, the offshore VSC is responsible for collecting energy generated by the offshore wind farm, it operates as a reference machine to set constant frequency, voltage amplitude and phase angle for the offshore network. Meanwhile, the onshore VSC controls the power flow to the onshore network by controlling the DC-link voltage and voltage amplitude at the connection point. A constant DC-link voltage indicates active power balance between onshore and offshore VSCs and ensures that the energy collected by the offshore VSC is transmitted to the onshore network.

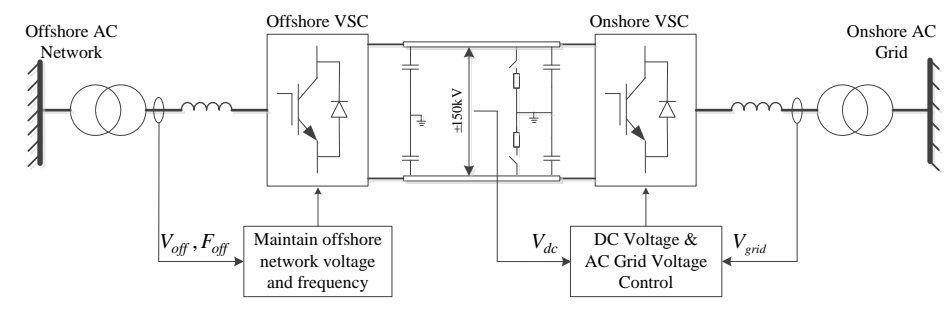

Fig. 3. Scheme of VSC-HVDC.

\subsection{Frequency control strategy}

As aforementioned, due to the frequency decoupling, the variation of onshore network frequency is not reflected on the offshore network. Therefore, a communication system may be needed to enable offshore wind turbines to contribute to power system frequency regulation. However, in this study, a simple PI control loop is added to the offshore VSC as shown in Fig. 4 (a) to realize fast and reliable transmission of onshore network frequency. The onshore VSC monitors the onshore network frequency $f_{\text {on }}$ and transmits it to the offshore VSC via the communication channel of VSC-HVDC itself. The offshore VSC then controls the offshore network frequency $f_{\text {off }}$ to vary with $f_{\text {on }}$. Therefore, with no complicated communication system, individual offshore wind turbines can detect the $f_{o n}$ by monitoring $f_{w f}$ and react to the excursion of $f_{\text {on }}$ rapidly.

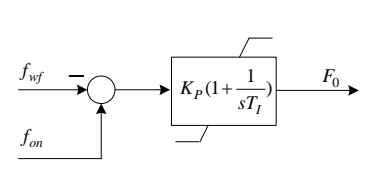

(a)

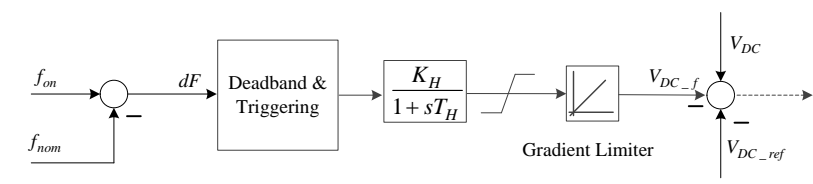

(b)

Fig. 4. Ancillary frequency control loop for VSC-HVDC: (a) for offshore VSC and (b) for onshore VSC.

Fig. 5 shows the equivalent circuits of the onshore VSC. As presented in [8], the mathematical model of the onshore VSC is in the synchronous rotating $d q$-reference frame, where the $d$-axis is aligned with the source voltage $V_{s}$, can be expressed as:

$$
\frac{d}{d t}\left[\begin{array}{l}
i_{s d} \\
i_{s q}
\end{array}\right]=\left[\begin{array}{cc}
-R / L & \omega \\
\omega & -R / L
\end{array}\right]+\frac{1}{L}\left[\begin{array}{l}
-v_{s d}+v_{c d} \\
-v_{s q}+v_{c q}
\end{array}\right]
$$




$$
\frac{d V_{d c}}{d t}=\frac{1}{C} I_{d c}-\frac{3}{2 C V_{d c}}\left(v_{c d} i_{s d}+v_{c q} i_{s q}\right)
$$

where $R$ and $L$ are the resistance and inductance of the phase reactor respectively, $\omega$ is the angular frequency. Considering that $P_{d c}=I_{d c} V_{d c}$ and $P_{a c}=3\left(v_{c d} i_{s d}+v_{c q} i_{s q}\right) / 2$, the dc-side mathematical model (2) can further be defined as:

$$
P_{a c}=P_{d c}-C \frac{d V_{d c}}{d t} \cdot V_{d c}
$$

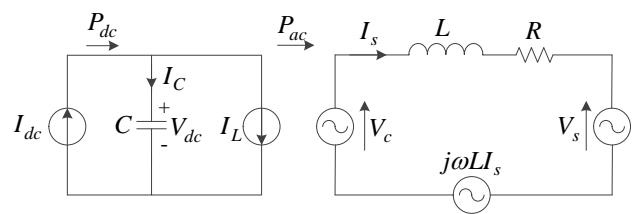

(a)

(b)

Fig. 5. Equivalent circuit of onshore VSC: (a) dc-side and (b) ac-side.

Assuming that the converter is lossless, thus a constant $V_{d c}$ means that the active power $P_{d c}$ entering the converter is equal to the active power $P a c$ leaving the converter in steady-state operations. However, as known that the capacitor is an energy storage element, a decrease in $V_{d c}$ would release the energy stored in the DC-link capacitor banks. The energy change $W_{c}$ and power change $p_{c}$ of a capacitor from $t_{1}$ to $t_{2}$ can be expressed as:

$$
W_{c}=C \int_{v_{d c}(t 1)}^{v_{d c}\left(t_{2}\right)} v_{d c} \cdot d v_{d c}, \quad p_{c}=\frac{d W_{c}\left(t_{1}, t_{2}\right)}{d t} .
$$

Thus, according to (3), it is possible for the onshore VSC to temporarily output more/less active power than the power collected by the offshore VSC, clearly, which can help the onshore network frequency regulation. Therefore, an ancillary control loop for frequency regulation is designed and added to the onshore VSC control as shown in Fig. 4 (b). When a variation of onshore network frequency occurs, it generates a signal to the onshore VSC control to change the DC-link voltage to release/take part of the energy stored in the capacitor banks.

\section{Simulations}

In this study, all system modeling and simulations are implemented on DIgSILENT PowerFactory platform. Simulations are carried out on the power system as shown in Fig. 6. The generators G1 and G2 are conventional synchronous generators with IEEE standard turbine-governor (gov_IEEEG1) and IEEE exciter (avr_IEEET1). Offshore wind farms rated at $270 \mathrm{MW}$, which is represented by an aggregated model, is connected to the main grid through VSC-HVDC. The loads are modeled as constant impedance.

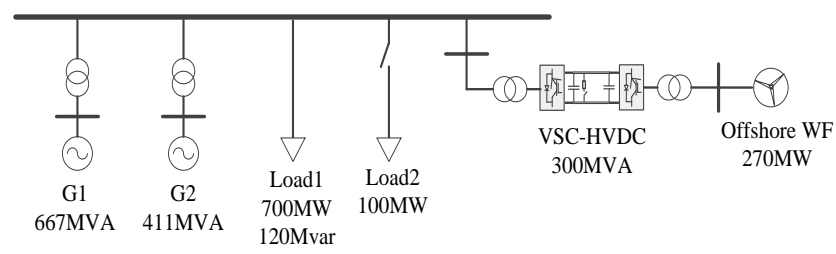

Fig. 6. Test power system.

In the initial state, the FCWTs operate with $10 \%$ de-loading curves and only Load 1 is connected to the grid. A sudden connection of Load 2 at $2 \mathrm{~s}$ is applied to cause a grid frequency drop. The communication time between onshore and offshore VSCs is assumed to be $300 \mathrm{~ms}$ and the lower limit of the DC voltage is set as $0.9 \mathrm{pu}$. 

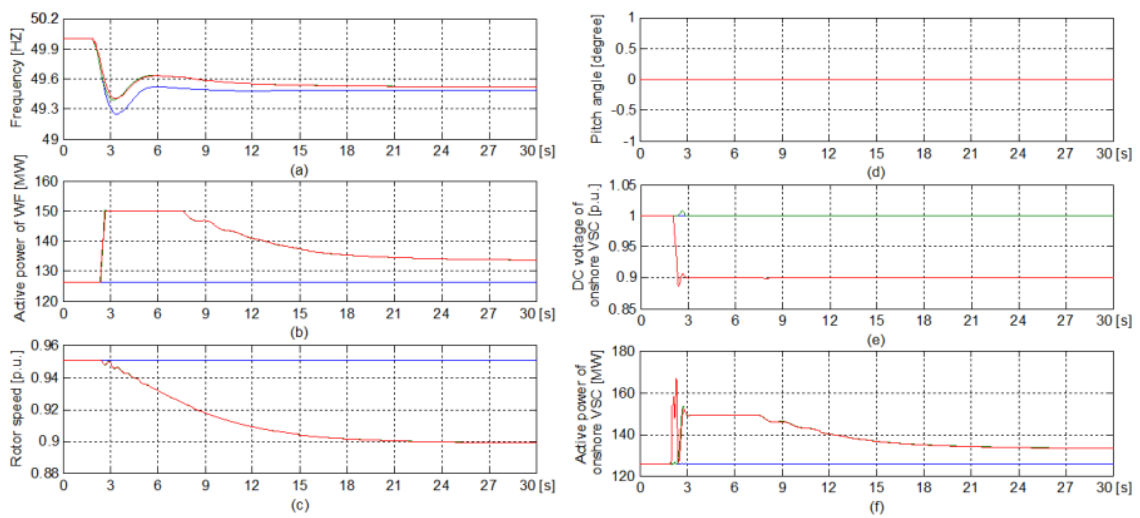

Fig. 7. Simulation results for low wind speed condition.
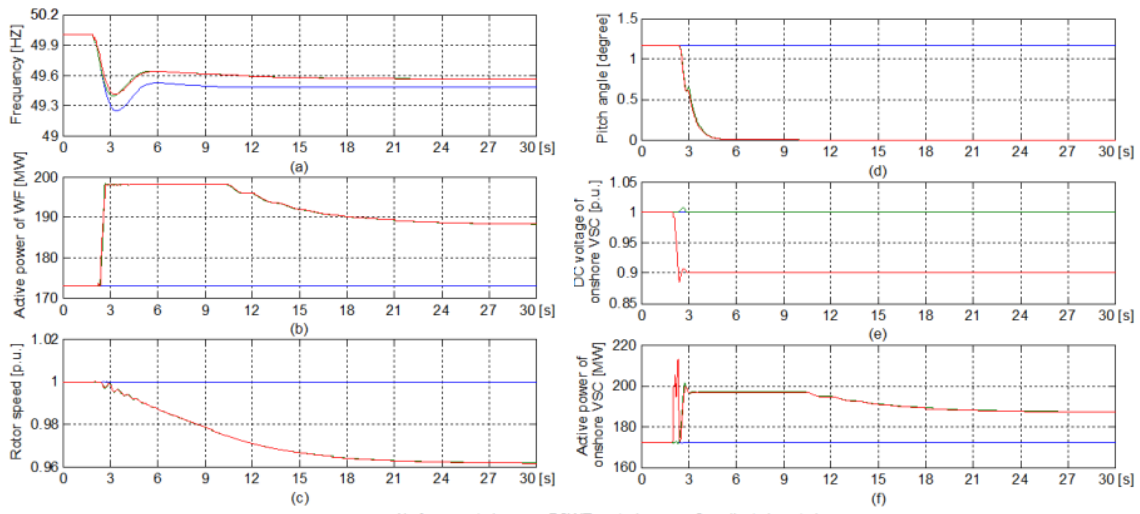

Fig. 8. Simulation results for medium wind speed condition.

\subsection{Low wind speed $(9 \mathrm{~m} / \mathrm{s})$}

In this case, the FCWTs operate in an over-speed mode to reserve power. Fig. 7 shows the simulation results of the system frequency and the dynamics of FCWTs and VSC-HVDC under three different conditions: 1) no any supplementary frequency controls; 2) frequency control of FCWTs only; 3) coordinated frequency control of FCWTs and VSC-HVDC.

Without ancillary frequency regulation of FCWTs and VSC-HVDC, the sudden connection of Load 2 results in a deep frequency drop but hardly affects the power generation of wind farms (see blue line in Fig. 7 (b) and (c). However, when the frequency controller of FCWT is activated, the kinetic energy and primary control reserve of FCWTs will be released via rotor speed reduction as a response to frequency deviation with a VSC-HVDC communication delay (see Fig. 7 (b) and (c)). Both the rate of change of the frequency (ROCOF) and the nadir of frequency are improved obviously. On this basis, if the frequency controller of VSC-HVDC is added, a further improvement on frequency response can be achieved as shown by red lines in Fig 6.

With the coordinated frequency control, VSC-HVDC releases the energy stored in capacitor banks at the instant of the increase of large power demand, and then FCWTs participate in frequency support after the energy-releasing of VSC-HVDC as shown in Fig. 7 (b) and (e). Fig. 7 (a) shows that though it has a negative impact on the frequency recovery process, the proposed coordinated control slows down ROCOF evidently and improves the frequency nadir.

\subsection{Medium wind speed $(10 \mathrm{~m} / \mathrm{s})$}

As discussed in Section 2.2, to implement $10 \%$ de-loading operation for this wind speed, rotor overspeed and pitch angle adjustment are both employed to FCWTs. Fig. 8 presents simulation results of 
system frequency, active power, rotor speed and pitch angle of FCWTs, and DC voltage and active power of the onshore VSC.

Before frequency excursion, the rotor speed in Fig. 8 (b) has reached 1.0 pu and the pitch angle in Fig. 8 (c) has also been increased. Both actions ensure the primary control reserve for FCWTs. When the frequency drop is detected, both rotor speed and pitch angle are reduced to allow FCWTs to generate more active power to support the frequency. Moreover, the inertial response is enhanced with implementation of the coordinated control as shown in Fig. 8 (f). Again, Fig. 8 (a) manifests the effectiveness of the proposed coordinated control on the improvement of ROCOF and nadir frequency.
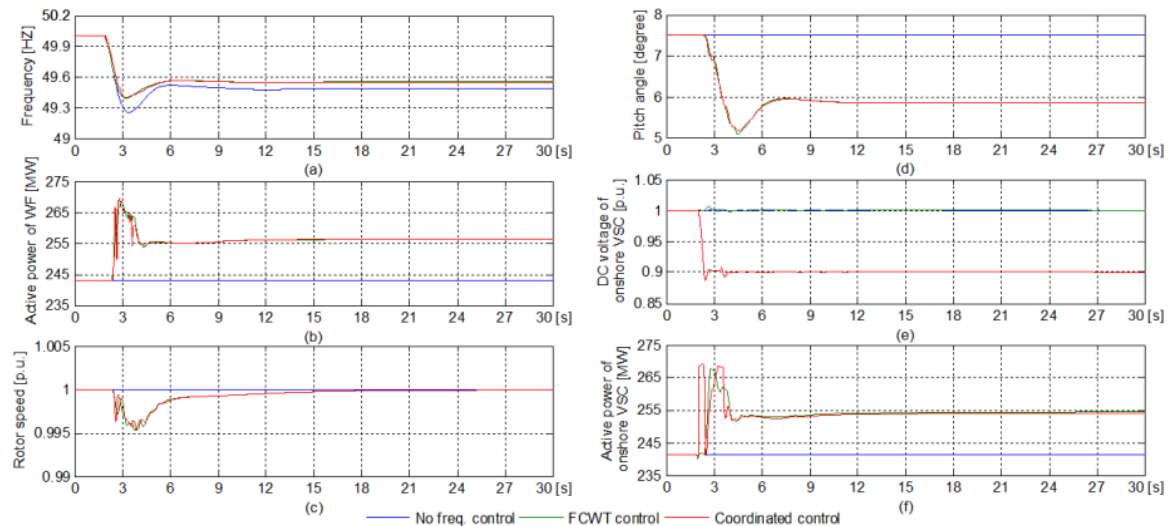

Fig. 9. Simulation results for high wind speed condition.

\subsection{High wind speed $(12.5 \mathrm{~m} / \mathrm{s})$}

Since this wind speed is higher than the rated speed, the wind turbine generator operates at its rated speed and the generation reserve is only achieved by the regulation of pitch angle. Simulation results for this condition are presented in Fig. 9.

Fig. 9 (a) shows that the proposed coordinated control method improves the ROCOF but deteriorates the frequency recovery compared to the sole frequency control of FCWTs, which is the same as observed in low and medium wind speed cases. As the speed of wind turbine rotors is at the rated value, the frequency regulation of FCWTs in this case is carried out by pitch angle adjusting as shown in Fig. 9 (d). However, if the FCWT's frequency controller is activated, Fig. 9 (c) shows that the rotor speed indeed reduces a bit when the FCWT senses the frequency drop, though it then returns to the rated speed soon. This is resulted from both the frequency controller and pitch angle controller of the FCWT. As a response to frequency drop, the frequency controller of the FCWT tends to slow down the generator rotor to extract kinetic energy. At the same time, the pitch angle controller is going to decrease the pitch angle to augment the turbine generation but keep the rotor speed constant. However, the blade pitch angle is controlled through the servo system, whose action is slower than power electronics. Therefore, the rotor speed goes down first and then speeds up to the rated value as shown in Fig. 9 (a).

Compared Fig. 9 (b) with (f), it is noticeable that under the conditions of no frequency control and FCWT frequency control only, the power curves in Fig. 9 (f) coincide with their counterparts in Fig. 9 (b). In other words, the active power transmitted by the onshore VSC equals to the active power generated by the wind farm minus all losses, for instance, cable loss and converter loss. However, under the condition of coordinated control (red line), the curve in Fig. 9 (b) is quite different from the curve in Fig. 9 (f) in the initial few seconds after the frequency drops, which indicates a power imbalance between offshore and onshore VSCs in that period. The explanation of this could be done by analysing Fig. 9 (e).

In this study, the rated power and DC voltage of VSC-HVDC are 300 MVA and $300 \mathrm{kV}$ respectively. That means that the VSC-HVDC can transmit as much as $300 \mathrm{MW}$ power with a constant $300 \mathrm{kV}$ DC link and is only to transmit maximum $270 \mathrm{MW}$ when the DC voltage is kept at $270 \mathrm{kV}$ (0.9 pu). In Fig. 9 (e), the DC voltage is controlled down to 0.9 pu rapidly and even causes an overshoot at around $2.5 \mathrm{~s}$ that lowers the transmitting capacity of VSC-HVDC to less than $270 \mathrm{MW}$. At almost the same time, the 
power output of the wind farm rockets close to 270 MW (Fig. 9 (b)). Consequently, these two factors lead to the power imbalance on the VSC-HVDC and DC voltage fluctuation, as shown in Fig. 9 (e).

\section{Conclusions}

This paper addresses the frequency regulation potential of VSC-HVDC and develops a practical control strategy, which is coordinated with the frequency control of FCWTs. The proposed coordinated control strategy succeeds in providing enhanced inertial response and primary frequency control. It solves the problem that offshore wind farms cannot provide inertial response in time because of the decoupling of the VSC-HVDC. The frequency control capability of VSC-HVDC is not affected by the generation of the offshore wind farm but heavily relies on its own configuration.

The offshore VSC controls the frequency of the offshore grid to vary with the onshore grid frequency variation so as to avoid complicate communication with individual wind turbines and effectively reduce the communication delay. The onshore VSC releases energy stored in HVDC capacitors by lowering down the DC voltage to provide virtual inertial response at the very instant of frequency drops. FCWTs operate in accordance with de-loading power curves so as to have the ability to provide primary frequency control. For a sudden frequency drop, the results demonstrate that the coordinated control achieves a better ROCOF and higher nadir frequency compared to frequency control of FCWTs only.

\section{Acknowledgements}

This work has been (partially) funded by Norwegian Centre for Offshore Wind Energy (NORCOWE) under grant 193821/S60 from Research Council of Norway (RCN). NORCOWE is a consortium with partners from industry and science, hosted by Christian Michelsen Research.

\section{References}

[1] Kundur P, Paserba J, Ajjarapu V, et al. Definition and classification of power system stability IEEE/CIGRE joint task force on stability terms and definitions. IEEE Transactions on Power Systems, 2004; 19(3):1387-1401.

[2] Lalor G, Mullane A, O"Malley M. Frequency control and wind turbine technologies. IEEE Transactions on Power Systems, 2005; 20(4):1905-1913.

[3] Morren J, De Haan SWH., Kling WL, et al. Wind turbines emulating inertia and supporting primary frequency control. IEEE Transactions on Power Systems, 2006; 21(1):433-434.

[4] Conroy JF, Watson R. Frequency response capability of full converter wind turbine generators in comparison to conventional generation. IEEE Transactions on Power Systems, 2008; 23(2)649-656.

[5] de Almeida RG, Castronuovo ED, Peas Lopes JA. Optimum generation control in wind parks when carrying out system operator requests. IEEE Transactions on Power Systems, 2006; 21(2):718-725.

[6] Zhang ZS, Sun YZ, Lin J, et al. Coordinated frequency regulation by doubly fed induction generator-based wind power plants. Renewable Power Generation, IET, 2012; 6(1):38-47.

[7] Jovcic D, Strachan N. Offshore wind farm with centralised power conversion and DC interconnection. Generation, Transmission \& Distribution, IET, 2009; 3(6):586-595.

[8] Xu L, Yao LZ, Sasse C. Grid integration of large DFIG-based wind farms using VSC transmission. IEEE Transactions on Power Systems, 2007; 22(3):976-984.

[9] Phulpin Y. Communication-free inertia and frequency control for wind generators connected by an HVDC-link. IEEE Transactions on Power Systems, 2012; 27(2):1136-1137.

[10] Akhmatova V, Nielsenb AH, Pedersenc JK, et al. Variable-speed wind turbines with multi-pole synchronous permanent magnet generators: part I: modelling in dynamic simulation tools. Wind Engineering, Jul. 2003; 27:531-548.

[11] Sun YZ, Zhang ZS, Li GJ, et al. Review on frequency control of power systems with wind power penetration. In: Proc. of 2010 International Conference on Power System Technology, 2010:1-8.

[12] Ma HT, Chowdhury BH. Working towards frequency regulation with wind plants: Combined control approaches. Renewable Power Generation, IET, 2010; 4(4):308-316. 